\title{
Relationships Between Markers of Inflammation and Muscle Mass, Strength and Function: Findings from the Hertfordshire Cohort Study
}

\author{
L. D. Westbury ${ }^{1}$ N. R. Fuggle ${ }^{1} \cdot$ H. E. Syddall ${ }^{1} \cdot$ N. A. Duggal ${ }^{2} \cdot$ S. C. Shaw ${ }^{1}$.

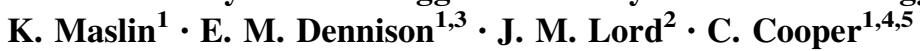

Received: 9 August 2017/Accepted: 19 October 2017/Published online: 3 November 2017

(c) The Author(s) 2017. This article is an open access publication

\begin{abstract}
We investigated the longitudinal relationships between inflammation markers and the following outcomes in a UK cohort study: appendicular lean mass (ALM); walking speed; level and change in grip strength; and sarcopenia defined by the European Working Group on Sarcopenia in Older People. Analyses were based on 336 community-dwelling older men and women (aged 59-70 years) who participated in the Hertfordshire Cohort Study (HCS). Inflammation markers were ascertained at baseline using enzyme-linked immunosorbent assay techniques and Bio-Plex Pro Assays. Grip strength was measured at baseline and follow-up [median follow-up time: 10.8 years (inter-quartile range 10.2-11.6)] and change in grip strength was ascertained using a residual change approach. At follow-up, ALM was ascertained using dualenergy X-ray absorptiometry, customary walking speed was measured and sarcopenia status was ascertained.
\end{abstract}

L. D. Westbury and N. R. Fuggle have contributed equally to the manuscript.

\section{Cooper}

cc@mrc.soton.ac.uk

1 MRC Lifecourse Epidemiology Unit, Southampton General Hospital, University of Southampton, Southampton SO16 6YD, UK

2 MRC-ARUK Centre for Musculoskeletal Ageing Research, Institute of Inflammation and Ageing, University of Birmingham, Birmingham, UK

3 Victoria University of Wellington, Wellington, New Zealand

4 NIHR Southampton Biomedical Research Centre, University of Southampton and University Hospital Southampton NHS Foundation Trust, Southampton, UK

5 NIHR Musculoskeletal Biomedical Research Unit, University of Oxford, Oxford, UK
Gender-adjusted linear and Poisson regression was used to examine the associations between inflammation markers and outcomes with and without adjustment for anthropometric and lifestyle factors. Higher C-reactive protein was associated $(p<0.04)$ with lower grip strength and accelerated decline in grip strength from baseline to follow-up. Higher cortisol was associated with lower ALM $(p<0.05)$. Higher interleukin-8 (IL-8) was associated with lower ALM $(p<0.05)$ and increased risk of sarcopenia [fully-adjusted relative risk per SD increase in IL-8: 1.37 (95\% CI 1.10, 1.71), $p=0.005]$. All associations were robust in fully-adjusted analyses. Inflammation markers were associated with measures of muscle mass, strength and function in HCS. Further work is required to replicate these associations and to delineate the underlying mechanisms.

Keywords Inflammation · Muscle $\cdot$ Sarcopenia $\cdot$ Strength · Adipokine · Interleukin

\section{Introduction}

Sarcopenia is an age-related syndrome characterised by loss of skeletal muscle mass and strength. It is a major contributor to the risk of physical frailty, functional impairment, poor health-related quality of life, and premature death in older people [1]. The prevalence varies [2] and it is associated with an estimated annual cost of $£ 2.5$ billion to the UK health economy [3].

Previous evidence supports the assertion that immunosenescence leads to a chronic, low-grade inflammatory state which contributes to cellular ageing, and an increased susceptibility to infection, autoimmunity and neoplasia [4]. A relationship may exist between inflammation and muscular 
ageing through a process of catabolism and skeletal muscle atrophy [5].

Interleukin-6 (IL-6), interleukin-1 receptor (IL-1R), tumour necrosis factor (TNF) and C-reactive protein (CRP) are primary elements in the inflammatory cascade which have been associated with disability [6], poor physical performance [7], reduced muscular strength [8] and muscle mass [9]. However, a recent meta-analysis of the relationship between three markers of inflammation and sarcopenia only showed a relationship with CRP but none with TNF or IL-6 [10]. This inconsistency highlights the need for further research which investigates the relationships between a wider range of inflammatory markers and sarcopenia.

The Hertfordshire Cohort Study (HCS) [11] provides an excellent opportunity to investigate these associations among a population-based cohort of community-dwelling older men and women from the United Kingdom.

The objective of this study was to investigate the longitudinal associations between a wide range of markers of inflammation and the following outcomes among HCS participants: appendicular lean mass (ALM), walking speed, level and change in grip strength, and sarcopenia defined according to the European Working Group on Sarcopenia in Older People (EWGSOP) consensus algorithm [1].

\section{Methods}

\section{The Hertfordshire Cohort Study}

The HCS comprises 1579 men and 1418 women born in Hertfordshire between 1931 and 1939 and who still lived there in 1998-2004 when they completed a baseline home interview and attended a research clinic for detailed characterisation of their socio-demographic, lifestyle and clinical characteristics; the study has been described in detail previously [11].

Smoking status, weekly consumption of alcohol and level of physical activity (Dallosso questionnaire [12]) were ascertained by a nurse-administered questionnaire at the home interview. Participants completed a food-frequency questionnaire from which a 'prudent diet' score was derived using principal components analysis; higher scores reflect healthier diets [13]. Details of all prescription and over-the-counter medications currently taken were coded according to the British National Formulary; the number of systems medicated was used as a marker of comorbidity.

Investigations conducted at the baseline clinic included measurement of height (using a Harpenden pocket stadiometer, Chasmors Ltd, London, UK) and weight (on a SECA floor scale, Chasmors Ltd, London, UK). Grip strength was assessed three times for each hand using a Jamar dynamometer; the highest measurement was used for analysis. Blood samples were taken and serum was aliquoted and stored at $-80{ }^{\circ} \mathrm{C}$.

Overall, 966 participants resident in East Hertfordshire underwent a dual-energy X-ray absorptiometry (DXA) scan at baseline. In 2004, 642 of them were recruited to a musculoskeletal follow-up study. In 2011, 591 were invited to participate in a further follow-up study; 443 agreed to participate [median follow-up time from baseline 10.8 years (inter-quartile range 10.2-11.6)] [14]. Grip strength and height were measured using the same protocol as at baseline, mean customary walking speed in metres per second was ascertained using the mean time from two $8 \mathrm{ft}$ gait speed tests, and ALM was derived using DXA (Lunar Prodigy Advanced Scanner, GE Medical Systems, UK).

The HCS analysis sample for this paper comprised 336 participants who: had attended the 2011 follow-up stage of HCS, had non-missing data for at least one of the inflammation markers considered; and had at least one nonmissing value for each of the outcomes. A flow diagram for the HCS analysis sample is presented in Fig. 1.

\section{Ascertainment of Baseline Inflammatory Markers}

Serum cortisol, dehydroepiandrosterone sulphate (DHEAS) and CRP levels were measured by enzyme-linked immunosorbent assay (ELISA) techniques using a commercial kit (IBL international, Hamburg, Germany) according to manufacturer's instructions. Plasma adiponectin (serum diluted 1:5000) and leptin (serum diluted 1:2000) were assessed separately by solid phase sandwich ELISA (R\&D Systems, Abingdon, UK). Data analysis was done using GraphPad Prism software (GraphPad Software Ltd, USA).

A multiplex based assay for the cytokines interleukin-10 (IL-10), interleukin-8 (IL-8), IL-6 and TNF (Bio-Rad Laboratories, Munich, Germany) was performed according to manufacturer's instructions. Data acquisition and analysis was conducted using Bio-Plex Manager software version 6.0.

\section{Derived Measures}

Height and weight were highly correlated $(r=0.43$, $p<0.001$ for men; $r=0.39, p<0.001$ for women); to avoid multi-collinearity problems, a sex-specific standardised residual of weight-adjusted-for-height at baseline was derived. Conditional change in grip strength was characterised by residuals obtained after estimating sex-specific linear regression models for grip strength at follow-up on grip strength at baseline with adjustment for individual follow-up duration; this measure of change is independent 


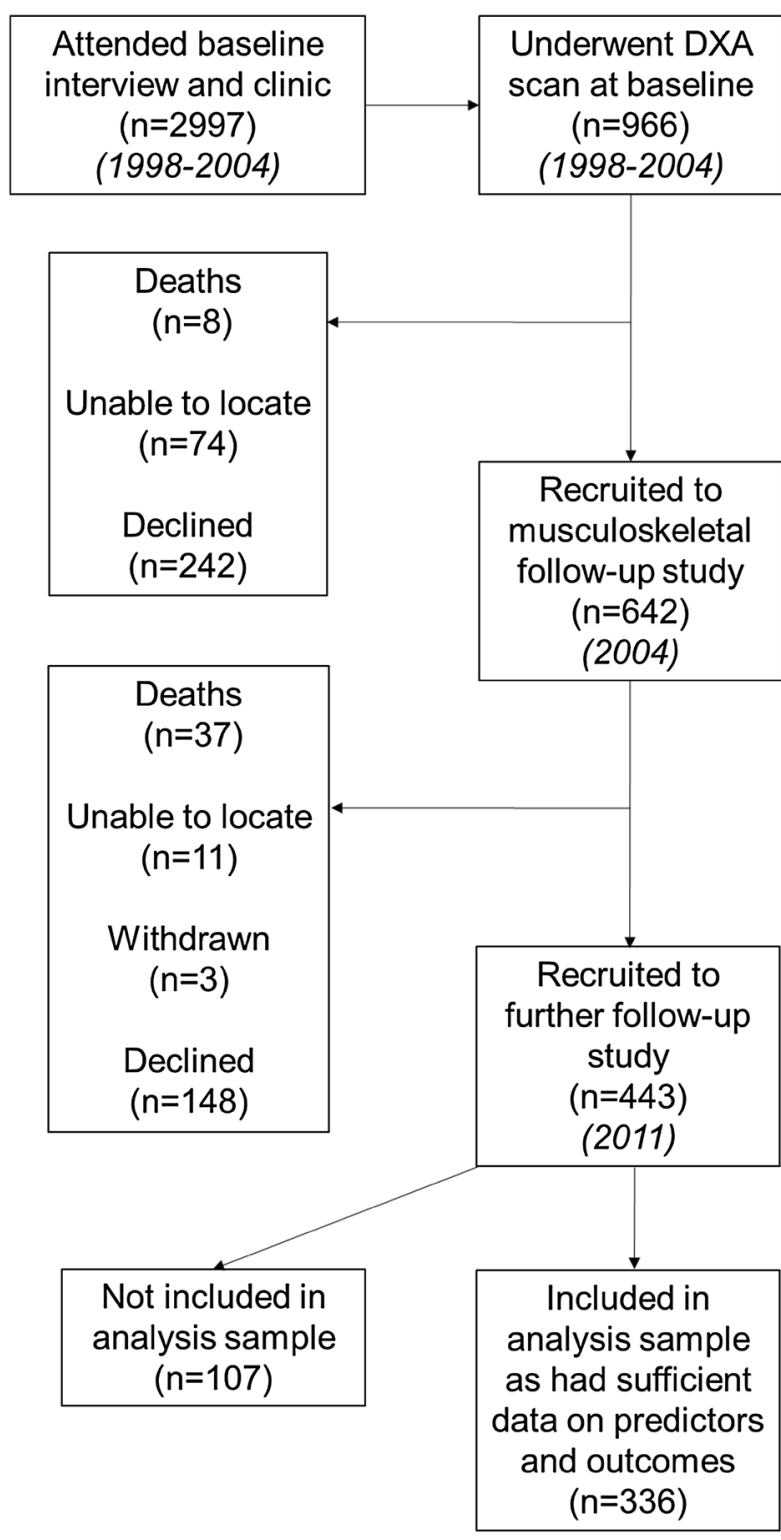

Fig. 1 Flow diagram for the Hertfordshire Cohort Study analysis sample

of baseline level. Appendicular lean mass index was calculated by dividing ALM $(\mathrm{kg})$ by the square of height $(\mathrm{m})$. Sarcopenia status at follow-up was derived using the EWGSOP sarcopenia algorithm [2] with the following cutpoints: ALM index $\left(\mathrm{ALM} / \mathrm{height}^{2}\right) \leq 7.23 \mathrm{~kg} / \mathrm{m}^{2}$ for men ( $\leq 5.67 \mathrm{~kg} / \mathrm{m}^{2}$ for women); grip strength $<30 \mathrm{~kg}$ for men $(<20 \mathrm{~kg}$ for women); and walking speed $\leq 0.8 \mathrm{~m} / \mathrm{s}$. Participants with slow walking speed or weak grip strength, and who also had low ALM index were classed as sarcopenic.

\section{Statistical Methods}

Summary statistics were used to describe the data. Apart from IL-8, all inflammatory markers were highly positively skewed and were log-transformed. Linear regression was used to examine the longitudinal association between each baseline inflammatory marker and the following continuous outcomes: walking speed, grip strength and ALM at follow-up; and conditional change in grip strength from baseline to follow-up. The association between baseline inflammatory markers and sarcopenia status at follow-up was examined using Poisson regression models with robust variance estimation to yield relative risks. Gender-adjusted and fully-adjusted models, which also accounted for gender, baseline age, follow-up time, height, weight-forheight, smoking history, alcohol consumption, prudent diet score and physical activity were implemented.

Sex-specific standard deviation scores were coded for inflammatory markers and outcomes and used in models. To maintain sample size, men and women were pooled and analyses were adjusted for gender; $p<0.05$ was regarded as statistically significant. Analyses were conducted using Stata, release 13 .

\section{Results}

\section{Participant Characteristics}

Characteristics of the 336 HCS participants who were included in the analysis sample are presented in Table 1. Mean (SD) age at HCS baseline was 63.8 (2.5) and 65.6 (2.7) years among men and women, respectively. Median (inter-quartile range) time from HCS baseline clinic to the 2011 follow-up was $11.6(11.1,11.9)$ years among men and $10.1(9.8,10.4)$ years among women. On average, men had higher ALM and faster walking speed compared with women. On average, men had much higher grip strength than women at both baseline and follow-up but experienced greater annual loss of grip strength (mean (SD) change in grip among men and women, respectively: $-0.71(0.48) \mathrm{kg} /$ year vs $-0.58(0.49) \mathrm{kg} /$ year $)$.

\section{Assessing Healthy Participant Effects in Analysis Sample}

Compared to the 2661 participants who attended the HCS baseline clinic but were not included in the analysis sample, both men and women in the analysis sample had higher self-reported activity (Dallosso questionnaire); diet quality was higher among women only $(p<0.003$ for both). However, smoking prevalence, alcohol consumption and the proportion who were of manual social class (classes 
Table 1 Characteristics of 336 participants from the Hertfordshire Cohort Study

\begin{tabular}{|c|c|c|c|}
\hline Mean (SD) & $\operatorname{Men}(n=186)$ & Women $(n=150)$ & Obs \\
\hline Age at HCS baseline clinic (years) & $63.8(2.5)$ & $65.6(2.7)$ & 336 \\
\hline Follow-up time (years) ${ }^{\mathrm{a}}$ & $11.6(11.1,11.9)$ & $10.1(9.8,10.4)$ & 336 \\
\hline Height $(\mathrm{cm})$ & $174.7(6.5)$ & $161.7(5.5)$ & 336 \\
\hline Weight (kg) & $80.8(11.0)$ & $69.2(12.2)$ & 336 \\
\hline $\operatorname{BMI}\left(\mathrm{kg} / \mathrm{m}^{2}\right)$ & $26.5(3.3)$ & $26.4(4.3)$ & 336 \\
\hline Ever smoked $^{\mathrm{b}}$ & $113(60.8 \%)$ & $50(33.3 \%)$ & 336 \\
\hline Alcohol consumer $(\geq 1 \text { unit per week })^{b}$ & $166(89.2 \%)$ & $86(57.3 \%)$ & 336 \\
\hline Prudent diet score & $-0.6(2.0)$ & $1.1(1.8)$ & 336 \\
\hline Dallosso physical activity score & $65.0(13.5)$ & $63.0(14.1)$ & 336 \\
\hline Number of systems medicated ${ }^{\mathrm{a}}$ & $1(0,1)$ & $1(0,2)$ & 336 \\
\hline $\mathrm{CRP}(\mathrm{mg} / \mathrm{L})^{\mathrm{a}}$ & $0.7(0.4,1.9)$ & $1.1(0.5,2.3)$ & 322 \\
\hline Adiponectin (ug/ml):Leptin (ng/ml) ratio $^{a}$ & $0.6(0.2,1.5)$ & $0.2(0.1,0.4)$ & 322 \\
\hline IL-10 $(\mathrm{pg} / \mathrm{ml})^{\mathrm{a}}$ & $7.0(5.9,10.6)$ & $7.2(5.9,10.6)$ & 334 \\
\hline IL-6 $(\mathrm{pg} / \mathrm{ml})^{\mathrm{a}}$ & $6.2(3.4,8.8)$ & $6.1(3.1,7.6)$ & 286 \\
\hline Cortisol $(\mathrm{ug} / \mathrm{ml})^{\mathrm{a}}$ & $0.10(0.09,0.12)$ & $0.09(0.08,0.10)$ & 331 \\
\hline DHEAS $(\mathrm{ug} / \mathrm{ml})^{\mathrm{a}}$ & $1.1(0.8,1.5)$ & $0.7(0.5,1.0)$ & 333 \\
\hline $\mathrm{TNF}(\mathrm{pg} / \mathrm{ml})^{\mathrm{a}}$ & $4.7(2.5,11.6)$ & $3.5(2.9,9.8)$ & 323 \\
\hline IL-8 $(\mathrm{pg} / \mathrm{ml})^{\mathrm{a}}$ & $7.5(5.4,10.5)$ & $6.9(5.8,9.2)$ & 268 \\
\hline Baseline grip strength $(\mathrm{kg})$ & $44.6(7.2)$ & $27.5(5.2)$ & 335 \\
\hline Grip change (kg/year) & $-0.71(0.48)$ & $-0.58(0.49)$ & 335 \\
\hline \multicolumn{4}{|l|}{ Characteristics at follow-up } \\
\hline Grip strength (kg) & $36.4(7.4)$ & $21.7(6.1)$ & 335 \\
\hline Appendicular lean mass $(\mathrm{kg})$ & $24.3(2.8)$ & $16.5(2.2)$ & 289 \\
\hline Appendicular lean mass index $\left(\mathrm{kg} / \mathrm{m}^{2}\right)$ & $8.05(0.75)$ & $6.42(0.72)$ & 289 \\
\hline Walking speed $(\mathrm{m} / \mathrm{s})$ & $0.79(0.17)$ & $0.74(0.18)$ & 314 \\
\hline EWGSOP sarcopenia $^{\mathrm{b}}$ & $12(7.4 \%)$ & $11(8.7 \%)$ & 289 \\
\hline
\end{tabular}

Grip change annual change in grip from HCS baseline to 2011 follow-up, EWGSOP European Working Group on Sarcopenia in Older People, Obs number of non-missing observations

${ }^{\mathrm{a}}$ Median (lower quartile, upper quartile)

${ }^{\mathrm{b}} n(\%)$

IIIM, IV and V from the 1990 Office of Population Censuses and Surveys (OPCS) Standard Occupational Classification (SOC90) unit group for occupation [15]) did not differ significantly $(p>0.05)$ between the two groups; this was the case among men and women.

Compared to the 107 participants who were invited to participate in the follow-up study in 2011 but were not included in the analysis sample, men in the analysis sample had higher self-reported activity $(p=0.01)$; there were no differences in other health behaviours or occupation class among men or among women.

\section{Associations Between Baseline Inflammation Markers and Muscle Mass, Strength and Function at Follow-Up}

Associations between baseline inflammation markers and the following outcomes are presented in Table 2 and Fig. 2: grip strength, walking speed, ALM and sarcopenia status at follow-up; and conditional change in grip from baseline to follow-up. In gender- and fully-adjusted analyses, higher CRP was associated with lower grip strength, accelerated loss of grip strength, and higher ALM. For example, an SD increase in CRP was associated with an average reduction in grip strength level of 0.21 (95\% CI $0.10,0.32)$ SDs. Higher CRP and lower adiponectin:leptin ratios were each associated with slower walking speed in gender-adjusted analyses only. In gender- and fully-adjusted analyses, higher cortisol and IL-8 were additional predictors of lower ALM. Higher DHEAS was only associated with lower ALM in gender-adjusted analysis.

\section{Associations Between Baseline Inflammation Markers and Sarcopenia at Follow-Up}

Lower adiponectin:leptin ratios and elevated levels of CRP, cortisol and IL-8 were associated with increased risk of sarcopenia as defined by the EWGSOP algorithm at follow- 
Table 2 SD difference $(95 \% \mathrm{CI})$ in outcome at follow-up per SD increase in inflammatory predictor

\begin{tabular}{|c|c|c|c|c|c|}
\hline \multirow[t]{2}{*}{ Outcome } & \multirow[t]{2}{*}{ Inflammatory predictor } & \multicolumn{2}{|l|}{ Gender-adjusted } & \multicolumn{2}{|l|}{ Fully-adjusted $^{\mathrm{a}}$} \\
\hline & & Estimate $(95 \%$ CI $)$ & $p$ value & Estimate $(95 \% \mathrm{CI})$ & $p$ value \\
\hline \multirow[t]{8}{*}{ Grip strength } & CRP & $-0.21(-0.32,-0.10)$ & $<0.001$ & $-0.21(-0.32,-0.10)$ & $<0.001$ \\
\hline & Ad:lep ratio & $0.05(-0.06,0.16)$ & 0.364 & $0.08(-0.03,0.19)$ & 0.135 \\
\hline & IL-10 & $0.05(-0.06,0.16)$ & 0.354 & $0.03(-0.07,0.13)$ & 0.529 \\
\hline & IL-6 & $-0.02(-0.14,0.10)$ & 0.771 & $-0.02(-0.13,0.09)$ & 0.725 \\
\hline & Cortisol & $-0.00(-0.11,0.10)$ & 0.930 & $0.00(-0.10,0.11)$ & 0.976 \\
\hline & DHEAS & $-0.04(-0.15,0.06)$ & 0.425 & $-0.02(-0.12,0.09)$ & 0.738 \\
\hline & TNF & $0.03(-0.08,0.14)$ & 0.543 & $0.03(-0.07,0.13)$ & 0.587 \\
\hline & IL-8 & $0.00(-0.12,0.12)$ & 0.945 & $0.06(-0.06,0.17)$ & 0.322 \\
\hline \multirow[t]{8}{*}{ Grip strength change } & CRP & $-0.15(-0.26,-0.04)$ & 0.006 & $-0.13(-0.24,-0.01)$ & 0.030 \\
\hline & Ad:lep ratio & $0.02(-0.08,0.13)$ & 0.686 & $0.02(-0.09,0.13)$ & 0.734 \\
\hline & $\mathrm{IL}-10$ & $0.07(-0.04,0.17)$ & 0.221 & $0.06(-0.05,0.16)$ & 0.306 \\
\hline & IL-6 & $0.07(-0.05,0.19)$ & 0.238 & $0.09(-0.03,0.21)$ & 0.122 \\
\hline & Cortisol & $0.08(-0.03,0.19)$ & 0.138 & $0.08(-0.03,0.19)$ & 0.132 \\
\hline & DHEAS & $0.03(-0.08,0.14)$ & 0.550 & $0.05(-0.06,0.16)$ & 0.386 \\
\hline & $\mathrm{TNF}$ & $0.05(-0.06,0.16)$ & 0.406 & $0.05(-0.06,0.16)$ & 0.374 \\
\hline & IL-8 & $0.01(-0.11,0.13)$ & 0.891 & $0.03(-0.09,0.15)$ & 0.647 \\
\hline \multirow[t]{8}{*}{ Walking speed } & CRP & $-0.18(-0.29,-0.07)$ & 0.001 & $-0.10(-0.22,0.01)$ & 0.085 \\
\hline & Ad:lep ratio & $0.15(0.04,0.26)$ & 0.008 & $0.08(-0.04,0.20)$ & 0.200 \\
\hline & IL-10 & $-0.05(-0.16,0.06)$ & 0.348 & $-0.07(-0.18,0.03)$ & 0.176 \\
\hline & IL-6 & $-0.01(-0.13,0.11)$ & 0.908 & $-0.00(-0.12,0.12)$ & 0.965 \\
\hline & Cortisol & $-0.02(-0.14,0.09)$ & 0.720 & $-0.06(-0.17,0.06)$ & 0.334 \\
\hline & DHEAS & $-0.01(-0.12,0.10)$ & 0.848 & $-0.01(-0.12,0.10)$ & 0.875 \\
\hline & TNF & $0.01(-0.10,0.12)$ & 0.898 & $-0.01(-0.12,0.10)$ & 0.894 \\
\hline & IL-8 & $-0.04(-0.16,0.08)$ & 0.478 & $-0.04(-0.16,0.08)$ & 0.541 \\
\hline \multirow[t]{8}{*}{ Appendicular lean mass } & CRP & $0.13(0.02,0.25)$ & 0.025 & $0.15(0.05,0.25)$ & 0.002 \\
\hline & Ad:lep ratio & $-0.11(-0.23,0.00)$ & 0.055 & $-0.05(-0.15,0.05)$ & 0.306 \\
\hline & IL-10 & $0.04(-0.07,0.16)$ & 0.480 & $0.03(-0.06,0.13)$ & 0.503 \\
\hline & IL-6 & $-0.00(-0.13,0.13)$ & 0.984 & $-0.07(-0.18,0.04)$ & 0.201 \\
\hline & Cortisol & $-0.12(-0.24,-0.00)$ & 0.042 & $-0.10(-0.20,-0.01)$ & 0.039 \\
\hline & DHEAS & $-0.13(-0.25,-0.01)$ & 0.032 & $-0.08(-0.18,0.02)$ & 0.107 \\
\hline & TNF & $-0.02(-0.14,0.09)$ & 0.700 & $-0.06(-0.15,0.04)$ & 0.227 \\
\hline & IL-8 & $-0.19(-0.32,-0.06)$ & 0.005 & $-0.11(-0.22,-0.00)$ & 0.043 \\
\hline \multirow[t]{8}{*}{ EWGSOP sarcopenia ${ }^{\mathrm{b}}$} & CRP & $1.37(0.90,2.08)$ & 0.144 & $1.77(1.08,2.90)$ & 0.023 \\
\hline & Ad:lep ratio & $0.86(0.61,1.21)$ & 0.387 & $0.62(0.44,0.87)$ & 0.006 \\
\hline & IL-10 & $1.06(0.74,1.51)$ & 0.761 & $0.99(0.74,1.34)$ & 0.969 \\
\hline & IL-6 & $1.44(0.78,2.68)$ & 0.246 & $1.27(0.71,2.28)$ & 0.416 \\
\hline & Cortisol & $1.39(1.08,1.79)$ & 0.011 & $1.35(0.95,1.91)$ & 0.092 \\
\hline & DHEAS & $1.12(0.75,1.68)$ & 0.576 & $1.11(0.74,1.64)$ & 0.619 \\
\hline & TNF & $1.32(0.87,2.02)$ & 0.194 & $1.31(0.89,1.94)$ & 0.167 \\
\hline & IL-8 & $1.57(1.35,1.84)$ & $<0.001$ & $1.37(1.10,1.71)$ & 0.005 \\
\hline
\end{tabular}

Separate regression models were fitted for each baseline inflammatory predictor

Sex-specific SD scores were derived for inflammatory predictors and continuous outcomes. Apart from IL-8, all inflammatory markers were logtransformed prior to standardising

Unadjusted estimates correspond to Pearson correlations between inflammatory markers and outcomes (apart from sarcopenia)

Change in grip strength from HCS baseline to follow-up was obtained using a residual change method to ensure grip change measure was independent of baseline grip level

Ad:lep ratio adiponectin:leptin ratio, EWGSOP European Working Group on Sarcopenia in Older People

Significant associations $(p<0.05)$ are in bold

${ }^{a}$ Regression models adjusted for the following characteristics at baseline: gender, age, follow-up time, height, weight-for-height residual, smoking history (ever vs never), alcohol consumption, diet quality and physical activity. Models for appendicular lean mass were not adjusted for weight-for-height residual and models for change in grip were not adjusted for follow-up time (follow-up time was already used to derive the grip change measure)

${ }^{\mathrm{b}}$ Estimates are relative risks per SD increase in baseline inflammatory predictors. Relative risks were derived using Poisson regression models with robust variance estimation 

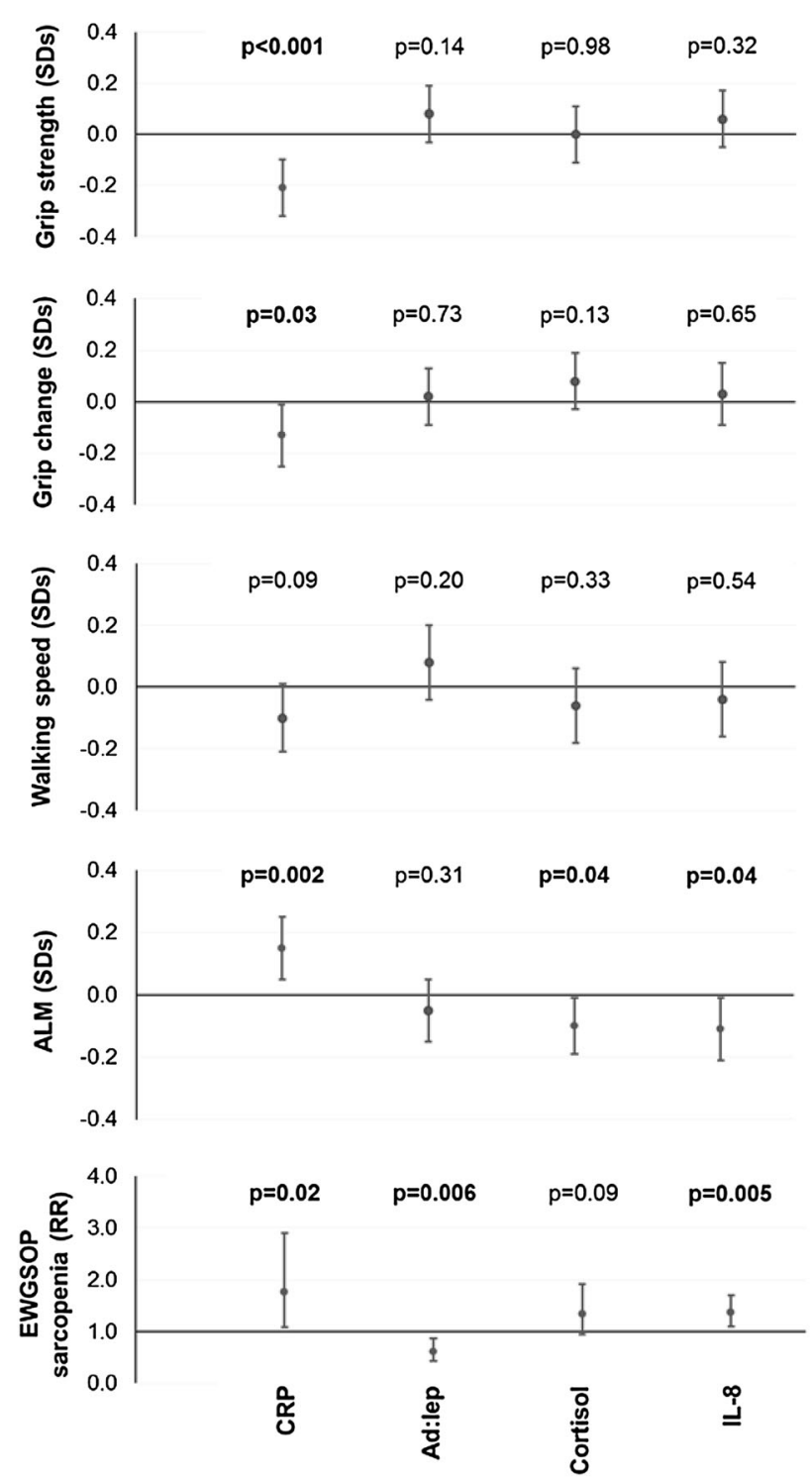

Fig. 2 SD difference $(95 \% \mathrm{CI})$ in outcomes per SD increase in inflammatory predictors. Ad:lep adiponectin:leptin ratio. Regression models adjusted for the following characteristics at baseline: gender, age, follow-up time, height, weight-for-height residual, smoking history (ever vs never), alcohol consumption, diet quality and physical activity. Poisson regression models with robust variance estimation were used for EWGSOP sarcopenia status to yield relative risks (RR). Models for ALM were not adjusted for weight-for-height residual and models for change in grip were not adjusted for followup time (follow-up time was already used to derive the grip change measure). Adjusted $p$ values are presented. Change in grip strength from HCS baseline to follow-up was obtained using a residual change method to ensure grip change measure was independent of baseline grip level. A positive estimate illustrates that a higher level of the inflammatory marker was associated with reduced loss of grip strength and a negative estimate reflects accelerated loss. Apart from IL-8, all inflammatory markers were log-transformed prior to standardising

up, although the strength of associations varied according to the other covariates included in the models.

\section{Discussion}

Using data from the HCS, we have shown that indices of inflammation are associated with each of the three components of sarcopenia; muscle mass, muscle strength and physical performance. The strongest associations were with CRP and were robust to adjustment for gender and lifestyle characteristics. Additional relationships were found with IL-8, a principal member of the inflammatory cascade, and cortisol, the most potent of the endogenous immunosuppressants. Fully-adjusted associations were similar when additionally adjusted for number of systems medicated (a marker of comorbidity) or steroid use. This study makes an important contribution to the published literature by examining the longitudinal associations between a wide range of inflammatory markers, cytokines and adipokines, with measures of muscle mass, strength, physical performance, and sarcopenia.

Our study has some limitations. First, selection bias may have affected our study at several stages. In common with many epidemiological studies, a healthy responder bias has been observed in HCS [11], however, baseline participants remained broadly comparable with participants in the nationally representative Health Survey for England [11]. In addition, sample attrition across the various waves of follow-up (possibly owing to mortality, morbidity and changes of address) could have resulted in further selection effects. However, we have described participant characteristics according to inclusion status across the study and found no major differences. Furthermore, because our analyses were internal, unless there was a systematic difference in the associations of interest among our study participants and those who were invited to take part in the study but did not, no major bias should have occurred. Secondly, although a relatively small sample size was used, the similarity between our findings and those of similar studies is encouraging [8, 16]. Thirdly, the markers of inflammation were measured at a single time point, which could be influenced by concurrent illnesses. However, due to the arrangement of the research clinics, it is unlikely that participants would have attended if acutely unwell or debilitated by long-term chronic disease. This is supported by that fact that the majority of the inflammation markers were within the normal range, suggesting that this was a largely healthy population. It can, therefore, be assumed that the inflammatory markers represent the inflammatory phenotype of the participant within the cohort as a whole. Fourthly, it is possible that the serum measurements of cytokine levels may not be indicative of the cytokine milieu at the level of the tissue of interest; muscle. Further in vivo, tissue-level studies are required to investigate this further. Finally, the availability of outcomes (other than 
grip strength) at baseline would have enabled investigation of the association between inflammation and change in muscle mass and function.

Our study also has many strengths. First, a residual change method was used to calculate a measure of change in grip strength which was independent of baseline grip strength and free from the effect of regression to the mean. Second, we examined the longitudinal associations between a broad panel of inflammatory markers and several defining components of sarcopenia with adjustment for a wide range of potential confounders (however, we cannot rule out the potential effects of unmeasured confounders). Finally, the HCS provides a strong foundation for research owing to: conduct of the fieldwork according to strict protocol by trained research nurses; detailed phenotyping of the cohort; and the continued management of the study by an experienced multidisciplinary team.

C-reactive protein is a consummate marker of inflammation, utilised ubiquitously in clinical practice and has been much studied in relation to many conditions. It is produced by the liver and rises up to 10,000 -fold in response to major tissue damage or severe infection [17]. However, outside of acute stimuli, the year to year, intraindividual variation in CRP is stable [18] with a significant association between baseline CRP and risk of vascular disease and mortality from cardiovascular disease, cancer and respiratory conditions [19]. This supports our use of a single measurement of inflammatory marker as a surrogate for overall, inflammatory phenotype. We found that a higher CRP was associated with lower grip strength, accelerated loss of grip strength, slower gait speed (in gender-adjusted analyses only), and sarcopenia (in fullyadjusted analyses only). Similar associations between reduced physical performance and grip strength and increasing CRP have been demonstrated in elderly Italian [8] and Dutch [20] cohorts. Further replication is provided by a previous study which demonstrated a graded association between increased CRP and reduced grip strength [21]. A similar, graded relationship between reduced CRP and faster gait speed has been seen in a multi-national study involving 64-74 year old participants from the North and South American continents [16]. This would support our finding of a negative association between CRP and walking speed.

The mechanism by which the association of CRP with parameters of muscle strength and function occurs is still under investigation though, a primed, pro-inflammatory environment, which would result in an increase in CRP as a marker of inflammation, is associated with the process of ageing, frailty and sarcopenia [22].

A raised CRP was also associated with greater ALM. This may seem counterintuitive, as a higher inflammatory burden would inhibit the anabolic processes involved in muscle tissue formation. However, if we consider that those with greater fat mass are also likely to have greater muscle mass (as demonstrated by ALM), a raised CRP would represent the increased inflammatory burden associated with adipose tissue. This is supported by the fact that CRP was not associated with ALM index when adjusted for whole body fat mass index in our sample (data not shown). Indeed, as adiposity increases, adipose tissue dysfunction can occur. Lipids leak into the circulation and deposit in muscle tissue leading to the localised production of reactive oxygen species and an inflammatory response (the latter of which will lead to a raised CRP). This can result in damage to mitochondria, muscle tissue damage, and thus, reduced muscle function.

Adipose tissue, besides its primary function as energy storage, also plays significant roles in the determination of the prevailing balance between pro- and anti-inflammatory states in the body as a whole. Adiponectin, an anti-inflammatory adipokine, inhibits nuclear factor $\kappa \mathrm{B}(\mathrm{NF} \kappa \mathrm{B})$ leading to reduced macrophage activation, reduced TNF and interferon gamma (IFN $\gamma$ ) and increases IL-10 and interleukin-1 receptor antagonist (IL-1RA). It is negatively associated with fat mass and leptin [23], and levels reduce with age [24]. On the other hand, leptin, a pro-inflammatory adipokine, reflects adipose mass [25] and is associated with the pro-inflammatory mediators; TNF, IL-6, interleukin-12 (IL-12) and activates natural killer lymphocytes [26]. In our study, we found that an increased adiponectin:leptin ratio was associated with faster walking speed (though this association was not robust beyond genderadjustment) and with reduced risk of sarcopenia. This is explained by the fact that a higher adiponectin:leptin ratio purports to represent a more anti-inflammatory adipokine profile, which would fit with a reduced propensity to muscle ageing and damage.

The negative associations of cortisol and IL-8 with ALM are simply explained.

IL-8 is a rife chemotactic agent for neutrophils and higher levels will represent a more primed, and active, innate immune response. Neutrophil dysregulation, including a reduced susceptibility to apoptotic signals and a more limited response to chemotactic agents, occurs with increasing age [27]. This is important in the process of inflammaging (a low-level state of inflammation as a result of an aged immune system) as delayed neutrophil transit and increased dysregulation leads to the leakage of matrix metalloproteinases and other enzymes leading to localised tissue damage. If this process was to occur within muscle, we would see a raised IL-8 in association with sarcopenia. Certainly, an active immune response has previously been associated with cachexia and frailty [22], so an association with sarcopenia would be aetiologically coherent. 
In order to counteract inflammaging, the body emboldens the process of 'anti-inflammaging' in the form of antiinflammatory cytokine production and cortisol release. Cortisol is the most potent, endogenous immunosuppressive agent and production can be stimulated by interleukin1 (IL-1), IL-6 and TNF via the hypothalamo-pituitary axis. However, as well as the anti-inflammatory effects [28], it has direct effects of bone resorption, gluconeogenesis and muscle catabolism. Higher levels of cortisol (as seen in the cortisol to DHEAS ratio) have been associated with significantly increased odds of frailty at 10-year follow-up [29]. Thus, the association we observed between higher cortisol and reduced ALM makes physiological sense and could be demonstrative of an increased anti-inflammatory, 'anti-inflammaging' response.

In summary, our findings highlight an important role for CRP, IL- 8 and other elements of immune function in the development of sarcopenia and its constituent components; muscle strength, muscle mass and physical function. Further work is required to delineate these relationships.

Acknowledgements The Hertfordshire Cohort Study was supported by the Medical Research Council [Grant No. MRC_MC_UU_12011/ 2, MRC_MC_UP_A620_1015] and University of Southampton UK. The funders played no role in the design, execution, analysis and interpretation of data, or writing of the study. We thank the men and women who participated in the Hertfordshire Cohort Study.

Author Contributions CC (guarantor) and JML designed the study. LDW conducted the statistical analysis and wrote the methodology and results. NRF conducted the literature review and wrote the background and discussion material. HES provided guidance regarding the statistical analyses. NAD conducted the laboratory assays. SCS and KM were the research assistant and research fellow of the Hertfordshire Cohort Study, respectively. EMD was the principal investigator of the Hertfordshire Cohort Study. All authors made substantial contributions to the manuscript and approved the final version.

\section{Compliance with Ethical Standards}

Conflict of interest L. D. Westbury, N. R. Fuggle, H. E. Syddall, N. A. Duggal, S. C. Shaw, K. Maslin, E. M. Dennison, J. M. Lord and C. Cooper declare that they have no conflict of interest.

Ethical Approval The baseline Hertfordshire Cohort Study had ethical approval from the Hertfordshire and Bedfordshire Local Research Ethics Committee and the follow-up had ethical approval from the East and North Hertfordshire Ethical Committees.

Human and Animal Rights All procedures performed in studies involving human participants were in accordance with the ethical standards of the institutional and/or national research committee and with the 1964 Helsinki declaration and its later amendments or comparable ethical standards.

Informed Consent All participants gave signed consent to participate in the study and for their health records to be accessed in the future.
Open Access This article is distributed under the terms of the Creative Commons Attribution 4.0 International License (http://crea tivecommons.org/licenses/by/4.0/), which permits unrestricted use, distribution, and reproduction in any medium, provided you give appropriate credit to the original author(s) and the source, provide a link to the Creative Commons license, and indicate if changes were made.

\section{References}

1. Cruz-Jentoft AJ, Baeyens JP, Bauer JM, Boirie Y, Cederholm T, Landi F, Martin FC, Michel JP, Rolland Y, Schneider SM, Topinkova E, Vandewoude M, Zamboni M (2010) Sarcopenia: European consensus on definition and diagnosis: report of the European Working Group on Sarcopenia in Older People. Age Ageing 39(4):412-423. doi:10.1093/ageing/afq034

2. Patel HP, Syddall HE, Jameson K, Robinson S, Denison H, Roberts HC, Edwards M, Dennison E, Cooper C, Aihie Sayer A (2013) Prevalence of sarcopenia in community-dwelling older people in the UK using the European Working Group on Sarcopenia in Older People (EWGSOP) definition: findings from the Hertfordshire Cohort Study (HCS). Age Ageing 42(3):378-384. doi:10.1093/ageing/afs 197

3. Westbury LD, Syddall HE, Sanchez-Santos MT, Dennison EM, Robinson SM, Cooper C (2017) Economic burden associated with sarcopenia: estimations from an English cohort study. Paper presented at the IOF, Florence

4. Michaud M, Balardy L, Moulis G, Gaudin C, Peyrot C, Vellas B, Cesari M, Nourhashemi F (2013) Proinflammatory cytokines, aging, and age-related diseases. J Am Med Directors Assoc 14(12):877-882. doi:10.1016/j.jamda.2013.05.009

5. Jo E, Lee SR, Park BS, Kim JS (2012) Potential mechanisms underlying the role of chronic inflammation in age-related muscle wasting. Aging Clin Exp Res 24(5):412-422. doi:10.3275/8464

6. Cesari M, Kritchevsky SB, Nicklas B, Kanaya AM, Patrignani P, Tacconelli S, Tranah GJ, Tognoni G, Harris TB, Incalzi RA, Newman AB, Pahor M (2012) Oxidative damage, platelet activation, and inflammation to predict mobility disability and mortality in older persons: results from the health aging and body composition study. J Gerontol Ser A Biol Sci Med Sci 67(6):671-676. doi:10.1093/gerona/glr246

7. Peterson MJ, Thompson DK, Pieper CF, Morey MC, Kraus VB, Kraus WE, Sullivan P, Fillenbaum G, Cohen HJ (2016) A novel analytic technique to measure associations between circulating biomarkers and physical performance across the adult life span. J Gerontol Ser A Biol Sci Med Sci 71(2):196-202. doi:10.1093/ gerona/glv007

8. Cesari M, Penninx BW, Pahor M, Lauretani F, Corsi AM, Rhys Williams G, Guralnik JM, Ferrucci L (2004) Inflammatory markers and physical performance in older persons: the InCHIANTI study. J Gerontol Ser A Biol Sci Med Sci 59(3):242-248

9. Beyer I, Mets T, Bautmans I (2012) Chronic low-grade inflammation and age-related sarcopenia. Curr Opin Clin Nutr Metab Care 15(1):12-22. doi:10.1097/MCO.0b013e32834dd297

10. Bano G, Trevisan C, Carraro S, Solmi M, Luchini C, Stubbs B, Manzato E, Sergi G, Veronese N (2017) Inflammation and sarcopenia: a systematic review and meta-analysis. Maturitas 96:10-15. doi:10.1016/j.maturitas.2016.11.006

11. Syddall H, Sayer AA, Dennison E, Martin H, Barker D, Cooper C (2005) Cohort profile: the Hertfordshire cohort study. Int J Epidemiol 34(6):1234-1242 
12. Dallosso HM, Morgan K, Bassey EJ, Ebrahim SB, Fentem PH, Arie TH (1988) Levels of customary physical activity among the old and the very old living at home. J Epidemiol Community Health 42(2):121-127

13. Robinson S, Syddall H, Jameson K, Batelaan S, Martin H, Dennison EM, Cooper C, Sayer AA, Group HS (2009) Current patterns of diet in community-dwelling older men and women: results from the Hertfordshire Cohort Study. Age Ageing 38:594-599

14. Dennison EM, Jameson KA, Edwards MH, Denison HJ, Aihie Sayer A, Cooper C (2014) Peripheral quantitative computed tomography measures are associated with adult fracture risk: the Hertfordshire Cohort Study. Bone 64:13-17. doi:10.1016/j.bone. 2014.03.040

15. Office of Population Censuses and Surveys (1990) Standard occupational classification, vol 1 structure and definition of major, minor and unit groups. HMSO, London

16. Sousa AC, Zunzunegui MV, Li A, Phillips SP, Guralnik JM, Guerra RO (2016) Association between C-reactive protein and physical performance in older populations: results from the International Mobility in Aging Study (IMIAS). Age Ageing 45(2):274-280. doi:10.1093/ageing/afv202

17. Gabay C, Kushner I (1999) Acute-phase proteins and other systemic responses to inflammation. New Engl J Med 340(6):448-454. doi:10.1056/nejm199902113400607

18. Emberson JR, Whincup PH, Morris RW, Walker M, Lowe GD, Rumley A (2004) Extent of regression dilution for established and novel coronary risk factors: results from the British Regional Heart Study. Eur J Cardiovasc Prev Rehabil 11(2):125-134

19. The Emerging Risk Factors C (2010) C-Reactive protein concentration and risk of coronary heart disease, stroke, and mortality: an individual participant meta-analysis. Lancet (Lond) 375(9709):132-140. doi:10.1016/S0140-6736(09)61717-7

20. Arts MH, Collard RM, Comijs HC, Naude PJ, Risselada R, Naarding P, Oude Voshaar RC (2015) Relationship between physical frailty and low-grade inflammation in late-life depression. J Am Geriatr Soc 63(8):1652-1657. doi:10.1111/jgs.13528
21. Norman K, Stobaus N, Kulka K, Schulzke J (2014) Effect of inflammation on handgrip strength in the non-critically ill is independent from age, gender and body composition. Eur J Clin Nutr 68(2):155-158. doi:10.1038/ejcn.2013.261

22. Wilson D, Jackson T, Sapey E, Lord JM (2017) Frailty and sarcopenia: the potential role of an aged immune system. Ageing Res Rev 36:1-10. doi:10.1016/j.arr.2017.01.006

23. Kalinkovich A, Livshits G (2017) Sarcopenic obesity or obese sarcopenia: a cross talk between age-associated adipose tissue and skeletal muscle inflammation as a main mechanism of the pathogenesis. Ageing Res Rev 35:200-221. doi:10.1016/j.arr. 2016.09.008

24. Voss SC, Nikolovski Z, Bourdon PC, Alsayrafi M, Schumacher YO (2016) The effect of cumulative endurance exercise on leptin and adiponectin and their role as markers to monitor training load. Biol Sport 33(1):23-28. doi:10.5604/20831862.1180173

25. Farr OM, Gavrieli A, Mantzoros CS (2015) Leptin applications in 2015: what have we learned about leptin and obesity? Curr Opin Endocrinol Diabetes Obes 22(5):353-359. doi:10.1097/med. 0000000000000184

26. Procaccini C, De Rosa V, Galgani M, Carbone F, La Rocca C, Formisano L, Matarese G (2013) Role of adipokines signaling in the modulation of $\mathrm{T}$ cells function. Front Immunol 4:332. doi:10. 3389/fimmu.2013.00332

27. Butcher S, Chahel H, Lord JM (2000) Review article: ageing and the neutrophil: no appetite for killing? Immunology 100(4):411-416

28. Giunta S (2008) Exploring the complex relations between inflammation and aging (inflamm-aging): anti-inflamm-aging remodelling of inflamm-aging, from robustness to frailty. Inflamm Res 57(12):558-563. doi:10.1007/s00011-008-7243-2

29. Baylis D, Bartlett DB, Syddall HE, Ntani G, Gale CR, Cooper C, Lord JM, Sayer AA (2013) Immune-endocrine biomarkers as predictors of frailty and mortality: a 10-year longitudinal study in community-dwelling older people. Age (Dordr) 35(3):963-971. doi:10.1007/s11357-012-9396-8 